\title{
Progress in caring for cancer patients in the scope of quality of life assessment
}

Ewelina Nawojska ${ }^{1}$, Anna Badowska-Kozakiewicz ${ }^{1}$, Aleksandra Czerw² ${ }^{2}$ Andrzej Deptała ${ }^{1}$

${ }^{1}$ Department of Cancer Prevention, Medical University of Warsaw, Poland

${ }^{2}$ Department of Health Economics and Medical Law, Medical University of Warsaw, Poland

Submitted: 11 October 2020

Accepted: 2 December 2020

Arch Med Sci

DOI: https://doi.org/10.5114/aoms/130651

Copyright $\odot 2021$ Termedia \& Banach

\section{Abstract}

Quality of life is assessed using standardised general or specific questionnaires. The use of such tools in scientific research makes it possible to determine how the patient functions in specific domains of life, and to measure the overall quality of life and health. A significant step forward in the approach to quality of life assessment is to also take into account factors relating to the patient's feelings and opinions as well as objective healthrelated factors. The patient is treated as an entity actively participating in the diagnostic and therapeutic process, whose subjective experiences are important. Research on quality of life assessment constitutes an essential element in the evaluation of the effectiveness of medical technologies. It also represents a new approach to formulating methods of dealing with patients, which is particularly crucial in care for chronically ill patients, including cancer patients.

Key words: assessment, cancer, oncology, quality of life, questionnaire.

\section{Introduction}

Along with the development of medical sciences, focused on extending life, preventing diseases, improving therapeutic methods, and reducing side effects, impairments and disability, we have observed an interest in the vital aspect of a sick person's well-being. Focusing on the subjective assessment of the patient's health, opinions, feelings, and sensations makes it possible to determine the declared level of quality of life (QoL). The QoL assessment is of particular importance in the care of patients diagnosed with chronic diseases, including cancer.

A holistic approach to caring for patients, which, in addition to the implementation of therapeutic goals, also includes non-medical goals, relating to the improvement of well-being and comfort of human functioning in various areas of life, is a significant advance in medical science.

\section{Definitions of quality of life}

Over the past few decades, we have observed a systematic increase in the importance and value of QoL assessment both in the social and medical sciences. It is the subject of many studies, part of everyday medical practice and a vital element in assessing the value of medical interventions.

\author{
Corresponding author: \\ Ewelina Nawojska \\ Department of Cancer \\ Prevention \\ Medical University \\ of Warsaw \\ 61 Żwirki i Wigury St \\ 02-091 Warsaw, Poland \\ E-mail: \\ enawojska@wum.edu.pl
}


When we look for forms to conceptualise the notion of QoL, it turns out that there is no universal definition of the term that provides a clear understanding. The concept of QoL has been included in the framework of many definitions that are not contradictory but based on various foundations and emphasise different aspects and areas of human functioning. They usually arise following fundamental questions about how people live, what is important to them, what the highest value is, how they assess their daily life from different perspectives, and whether it is satisfying to them.

Considerations on the QoL in the context of health and disease have resulted in the formulation of a narrower, more exact term - health-related quality of life (HRQoL). A valuable part of scientific achievements related to this issue is Schipper's studies, which in the 1990s defined HRQoL as "the influence of a disease and its treatment perceived by the patient" [1]. According to the author, a person makes an individual assessment regarding the impact of disease and the therapeutic process on their functioning in four basic areas: physical, mental, social and in the sphere of somatic sensations. The author emphasises the importance of a holistic view of QoL, taking into account the medical aspect, including physical condition, mobility, mental state and somatic sensations, as well as the non-medical aspect regarding functioning in the family, work, and society.

According to the World Health Organization, $\mathrm{HRQOL}$ is "the direct and indirect influence of disease and the treatment process, experienced and perceived by a patient" [2]. The approach emphasises the impact of health on subjective experiences, feelings, and assessments regarding the patient's functioning in various aspects of life.

Modern definitions of QoL include numerous aspects of human life, such as health, well-being, security, relationships with others, life in society, the opportunity to learn, creative activities, helping others, situation and material comfort, and leisure [3].

Quality of life is an important value for patients, but very difficult to measure and categorise. This concept is considered extremely subjective, which means that its interpretation and assessment depend on the mental state of an individual, their character, personality, preferences, current ailments, or accepted system of values. When assessing the QoL, it is essential to distinguish the patient's subjective feelings, i.e. their perception of the QoL, from an objective, medical point of view, premises about their health, regarding the type of illness, possible symptoms, consequences, or side effects of therapy [4]. Furthermore, QoL assessment is dynamic, i.e. it changes over time due to the patient's adaptation to the disease [5].

\section{Quality of life in cancer}

The measurement and assessment of QoL in cancer patients is a difficult and complicated activity due to the complex and long-term nature of the disease. The measurement of QoL in cancer patients is undoubtedly influenced by stress, which occurs already at the stage of diagnosis. Cancer patients experience physical stress related to pain and suffering; emotional stress manifested in irritation, anxiety, anger or depressive states; and social stress associated with the impossibility of performing social roles, the need for isolation and a feeling of rejection.

When ill, patients are forced to develop a specific, proper way of accepting and adapting to cancer. It is a long-term process aimed at eliminating the feeling of mental and emotional discomfort and restoring the fullest possible internal balance in the face of a new life situation, which is a serious disease. Adopting an appropriate method is essential for current health and improvement of the life situation, as well as for long-term health consequences. According to Moorey and Greer (1989), there are five main styles of adaptation to cancer: brave (fighting, active, optimistic, not giving up), coping (using defence mechanisms - denying, avoiding, minimising the problem), adopting a fatalistic attitude (maintaining calmness and self-control, accepting fate, convinced that "what will be, will be"), adopting an attitude of passivity and hopelessness (frightened, depressed), feeling anxious (overwhelmed by trifles, adopting hypochondriac attitudes) [6].

Patients adopting one of the first three strategies have a chance to adapt well to cancer and cope with the associated physical and mental stress. The next two styles, which are the worst adaptation methods, significantly reduce the QoL.

The development of a specific coping style depends on several factors: the type and severity of the disease, therapeutic method used, age, level of support and care provided to the patient, their character, temper, family relationships, and even professional and economic situation.

In the assessment of psychological adaptation to cancer, Czerw et al. identified coping strategies adopted by cancer patients [7]. Patients of the Oncology and Haematology Clinic of the Central Clinical Hospital of the Ministry of Interior and Administration in Warsaw participated in the study. Their level of adaptation to the disease was measured using the Mental Adaptation to Cancer Scale Mini-MAC. Each of the four dimensions of the scale - fighting spirit, positive re-evaluation, helplessness-hopelessness, and anxiety 
- were juxtaposed with demographic and socioeconomic characteristics to investigate the relationships between them. The results obtained from the study indicated that cancer patients demonstrate significant behaviours that allow for the determination of the coping strategies they adopted. People diagnosed with cancer demonstrate stronger constructive behaviour, in the areas of fighting spirit and positive re-evaluation than destructive - helpless-hopelessness and anxiety. It has been observed that the dominant behaviours in the area of helplessness-hopelessness are more common in men than in women, which implies that men suffering from cancer more often adopt a passive attitude, lose their fighting spirit, suffer from depression, doubt and resignation. It has also been proved that people with metastases, compared to patients without, demonstrate stronger behaviours typical of the area of helplessness-hopelessness. The most common attitudes in the group of patients with metastases are depression, doubting recovery, and eventually conceding defeat in the fight for health and life. The same destructive model of coping is more often observed among patients who are pensioners than in working people. Pensioners demonstrate behaviours that show a sense of powerlessness and helplessness. The results also showed that cancer patients demonstrate the fighting spirit strategy for the shortest time. They adopt an active, fighting attitude focused on the task and goal of overcoming the disease. However, with time, fatigue, exhaustion, and resignation appear, and the desire to overcome the disease subsides. The study showed that cancer patients use different coping strategies depending on demographic and socio-economic factors.

It is worth emphasising that patients' actions and efforts to better adapt to the disease can be supported by shaping and developing individual resources, including positive thinking, self-esteem, resourcefulness, strengthening the sense of control, and mobilisation in difficult situations [8].

When appreciating the importance and nature of an approach to the cancer experience, we observe that one of the challenges of modern medicine is - in addition to improving general health and extending life - ensuring an adequate level of quality in human functioning in the physical, mental and social sphere. For this purpose, it is necessary to explore the subjective dimension as closely as possible, which is the assessment of health and QoL perceived from the viewpoint of the patients themselves.

\section{Usefulness of HRQOL research}

Research on patients' QoL provides insight into the nature of the disease from a non-clinical perspective. It allows us to take into account, appreciate and focus on personal views of patients regarding their well-being, sensations, and emotions, and thus provide a new view on the disease process. It helps us learn and assess the mechanisms of influence of the disease on human functioning in various spheres of life. Quality of life assessment applies in the following areas.

\section{Medical practice}

Quality of life assessment is an extremely valuable element of the entire patient care process. It supplements the medical premises with personal feelings, observations, and opinions of the patient about their difficult situation, i.e. experiencing the disease. It helps identify areas that are the "weakest links" among the functioning spheres of an individual, in which he/she requires the greatest support. Such action allows more accurate choices to be made in the complex process of patient care [9].

\section{Doctor-patient relations}

Medical personnel's understanding of how the disease affects the quality of human life can become an incentive to improve communication methods tailored to the individual needs of patients, as well as increasing the feeling of empathy, which translates into a significant improvement in the relationship between doctors and patients.

Facts about the patient's life and experience of the disease are often insignificant from the viewpoint of medical personnel; however, in the patient's opinion, they are of fundamental importance and can significantly affect health. Research on QoL is also an impulse to continually search for alternative therapy methods, taking into account the patient's predispositions, life situation, mental state, personality traits, needs and expectations [10].

Thanks to such procedures, doctors have a greater sense of their work and professional fulfilment, and patients are provided with more comprehensive care. By clearly emphasising and appreciating the importance of assessing the QoL, patients become a partner in the complex and long-term process of treatment and recovery.

\section{Pharmacoeconomic analyses to assess treatment effectiveness}

Assessment of the effectiveness of the therapy used, including traditional health indicators and analysis of the impact of therapy on symptom control, as well as the frequency and type of complications, is supplemented with an assessment of the patient's QoL. Information obtained directly from the patient is relevant to determine their 
subjective concerns, ailments and needs. It implies that the subject of medical interest is not only to prolong life but also improve well-being and bring the level of a patient's QoL closer to the level before the onset of the disease.

In the face of continually growing health needs, which are met by new medical technologies, one can observe a constant increase in financial expenditure on health care. Therefore, a question arises as to whether specific procedures and technologies contribute to achieving a measurable health effect, and whether they correlate with a higher level of patient satisfaction and wellbeing - and thus a sufficiently high level of QoL. As a result, QoL assessment is becoming increasingly crucial in pharmacoeconomic analyses conducted to assess treatment effectiveness.

The QALY measure (quality-adjusted life year) is used to compare alternative therapeutic methods. Using the QALY measure, it is possible to express, in measurable categories, additional years of life obtained through the use of specific medical interventions, taking into account the accompanying QoL described on a scale of 0-1. To calculate QALY, these two values are multiplied [11].

\section{Health policy}

The QoL assessment can be part of the evaluation of changes introduced under health and social policy. It allows us to check whether the implemented modifications have provided the intended effect, i.e. whether they correlate with a better QoL for people in the population covered by a given programme [12].

\section{Questionnaire methods to assess the quality of life of cancer patients}

Progress in assessing QoL is associated with the introduction of questionnaire methods that allow for a thorough analysis of the mental state, level of satisfaction and well-being of cancer patients, in addition to their physical condition. Thanks to their use, the researcher obtains a quantitative result that can be easily compared with the results obtained from research on QoL conducted on different groups of patients. In addition, conducting research based on questionnaire methods is not time-consuming and is relatively convenient.

Questionnaires enable the assessment of various aspects of life, where the answers are assigned specific partial values. Then, they are added to values defining the level of QoL in the main areas of human functioning. The total score is a measure of the overall level of QoL of the studied patient.

There are two basic groups of the most commonly used tools for measuring QoL. General (ge- neric) questionnaires are designed to study the QoL in a broad, general range, in large and diverse populations of healthy and sick people, with various health problems. Thanks to this, one can make any comparisons within different groups of people. However, the general nature of the tools constitutes a limitation - they show low sensitivity in identifying small, but crucial changes in QoL, from the patient's point of view because the aspect which is mainly affected by the disease may be insufficiently represented by questionnaire questions. The second group comprises specific questionnaires, which are designed to measure QoL in particular diseases (e.g. breast cancer, colorectal cancer, lymphoma) or groups of diseases (e.g. cardiovascular diseases). Questions contained in these types of tools only concern aspects that are relevant to a specific target group. For this reason, they are characterised by greater sensitivity in identifying changes in the level of QoL than generic questionnaires [13].

The defined research goal dictates the choice of a specific tool. A good practice is to use a general tool or a combination of a general and specific tool to assess the studied phenomenon more comprehensively [14].

In the mid-twentieth century, the general condition and QoL of people diagnosed with cancer were assessed only on the basis of their physical limitations - e.g. using the Karnofsky Performance Index. The scale, determining the degree of functional status, ranges from 0 to 100 , where 0 means death, and 100 means a normal condition, no ailments, or symptoms of disease [15]. Since the 1990s, significant progress in the approach to assessing QoL has been observed, which allowed for a holistic approach to this research issue, while respecting its multidimensional and interdisciplinary nature. The majority of measurement tools used at the time were designed in such a way as to help learn and analyse additional in dicators for the overall assessment of QoL, such as ailments, work of the senses, the performance of social roles, cognitive functioning, emotional and mental state, and self-service of the patient.

The following research tools can be distinguished among the most commonly used methods for assessing the QoL of cancer patients.

\section{SF-36 questionnaire (Short Form (36) Health Survey)}

The SF-36 questionnaire was developed by the research organisation RAND Corporation, as part of a multi-year, multi-centre study, aimed at explaining differences in patient outcomes (Medical Outcomes Study (MOS)). The SF-36 tool, in its original version, has been made available for free use by RAND Corporation. In addition, a group of scien- 
tists conducting the MOS study developed a commercial version of the SF-36 questionnaire [16]. Currently, the research uses the second version of the tool (SF-36v2), available in over 170 language versions.

The SF-36 tool is an instrument designed to be completed by patients and used to measure the subjective health assessment of healthy people or patients with various diseases. It contains 11 questions with 36 statements. They are assigned to eight areas in which the QoL is assessed: physical functioning (PF), role physical $(\mathrm{RP})$, mental health $(\mathrm{MH})$, role emotional (RE), social functioning (SF), bodily pain (BP), vitality (VT), general health $(\mathrm{GH})$.

Moreover, health transition ( $\mathrm{HT})$ is assessed, i.e. current health in comparison to health a year earlier [17].

The QoL indicator is calculated by adding up points from all eight scales, which allows for an overall assessment of health.

The indicators can be summed up into two general domains related to the physical health summary (PHS) - as the average of points from the PF, RP, BP and GH scales - and the mental health summary (MHS) - as the average of points obtained in the scope of the MH, RE, SF and VT scales. The highest number of points obtained means the lowest degree in the QoL assessment, while the lowest value is associated with the highest level of QoL [17].

\section{WHOQOL-BREFF Questionnaire (World Health Organization Quality of Life - BREFF)}

In 1991 the World Health Organization initiated the WHOQOL (World Health Organization Quality of Life) project, which developed the WHOQOL-100 questionnaire - a universal instrument for measuring the overall assessment of QoL, which can be used in international and intercultural settings. It includes 100 questions evaluated on a five-point scale, which are assigned to $6 \mathrm{ma}$ jor domains, covering 24 aspects of QoL. Furthermore, it contains a general subscale that consists of 4 questions related to the overall, individual assessment of health and QoL [9].

Based on the WHOQOL-100, a shortened version of the tool was created - the WHOQOL-BREF questionnaire. It is used to measure QoL for both sick and healthy people. It consists of 26 questions, assigned to the following 4 domains, in which the functioning of an individual is studied: physical domain (pain and discomfort, sleep and rest, energy and fatigue, daily activity, mobility, ability to work, use of medications and use of medical assistance), psychological domain (experiencing positive and negative feelings, satisfaction with appearance, self-esteem, personal beliefs, cognitive skills), social domain (social relations, social support, sexual activity) and environmental domain (a sense of freedom, security, the nuisance of the physical environment, financial resources, availability and quality of health and social care, home environment, the opportunity for rest and recreation, opportunity to acquire new skills and obtain information, communication) [18].

Answers to questions are given on a five-point scale. Additionally, the questionnaire contains two questions on the subjective, overall assessment of QoL and health, which are subject to a separate analysis.

\section{EQ-5D Questionnaire (Euro Quality of Life Questionnaire)}

The EQ-5D questionnaire was developed by the EuroQol Group in 1990. It is a standardised tool for measuring the overall assessment of QoL, applicable to people over 12 years of age [19]. It is a universal tool, used in the field of health assessment of individuals, groups of patients and populations. The tool is simple and accessible. It was designed such that the respondent can make a quick self-assessment without assistance from a researcher. It is available in electronic and paper form. It has been translated into over 200 language versions.

The EQ-5D questionnaire can be widely used, in less complex studies assessing health and QoL as well as in multivariate studies - in combination with other tools, it is used in clinical studies and for the preparation of pharmaco-economic analyses [20].

EQ-5D consists of two parts: description and health assessment.

In the descriptive module, health is measured in five dimensions (5D): mobility, self-care, daily activity, pain/discomfort, anxiety/depression.

Respondents make self-assessments in terms of functioning in these areas, using a three-point (EQ-5D-3L) or five-point (EQ-5D-5L) scale. A specially dedicated version of EQ-5D-Y was developed for young people, with wording appropriate for the younger age group.

Results on the descriptive scale are presented in the form of a five-digit code - each dimension is assigned a digit corresponding to the answer given, where 1 means no difficulty, 2 means occurrence of difficulties, and 3 means serious difficulties. The result of 11232 means that the person has no problems with mobility and in the field of self-care, he/she experiences difficulties during everyday activities, the individual has moderate symptoms of anxiety/depression, and pain is a serious problem.

In the second part, the respondent assesses their health on a visual analogue scale (EQ-VAS), 
with endpoints 0 and 100, where 0 means "the worst imaginable health" and 100 "the best imaginable health" [21].

\section{NHP Scale (Nottingham Health Profile)}

The NHP questionnaire was developed by Hunt and McEwen, as a tool to measure health problems described by a patient and to assess the impact of these difficulties on their functioning in everyday life. The method has been functioning since 1986 [22] and is widely used to subjectively assess the QoL of people with specific health problems.

The NHP questionnaire consists of two parts. The first is composed of 38 statements related to six main areas: pain, energy, sleep, mobility, emotional reactions, and social isolation. The second part consists of 7 individual elements referring to the following areas of life affected by the identified difficulties: social life, work, family life, housework, sex life, interests and hobbies as well as holidays and free time. Respondents can provide 'yes' or 'no' answers to each statement. The answers are scored depending on the significance of health problems. A maximum of 100 points can be obtained in each section - the higher the score, the greater the intensity of problems the patient experiences in functioning in specific areas of life, which translates into a lower level of QoL [22].

\section{Spitzer's Quality of Life Index}

The scale was developed in the 1980s by a research group operating under the direction of Spitzer, tasked with assessing the QoL in cancer patients. It was assumed that the survey would be completed by a doctor, based on knowledge of a given patient; however, over time, it turned out that this instrument could also be used for self-assessment by patients themselves. It is characterised by simplicity, ease of implementation and speed of calculation of results. Moreover, it perfectly differentiates the study group in terms of type and stage of the disease.

The tool consists of 15 statements assigned to 5 main areas: activity, daily living, health, support of family and friends, outlook.

Each element of the QoL assessment can be assigned a 0-2 answer. The analysis is performed by adding up the results for each area separately or together for the entire scale [23].

\section{QLQ-C30 (Quality of Life Questionnaire - Core 30$)$}

QLQ-C30 questionnaire was developed by the Quality of Life Group (QLG), established in 1981 as part of activities of the European Organisation for Research and Treatment of Cancer
(EORTC), the objective of which is aimed at leading, initiating, developing and coordinating cancer research in Europe. QLQ-C30 is a core questionnaire for the general assessment of various aspects defining the level of QoL in the studied group of cancer patients. It has been translated and approved in over 110 language versions. Its use is protected by copyright. However, the group may give consent to the use of the questionnaire free of charge in research to achieve its scientific goals. Currently, the latest version, QLQ-C30 3.0, is recommended for use in research [24].

Given the general nature and limitations of the QLQ-C30 questionnaire, QLG assumed that the measurement of the QoL in patients with different types of cancer would be conducted using the core tool QLQ-C30 with additional specific modules that have been developed to more accurately assess aspects affecting the level of QoL in patients with specific types of cancer.

The QLQ-C30 questionnaire consists of 30 questions, which are assigned to the following areas. Five scales assess the functional condition: physical functioning (PF), role functioning (RF) emotional functioning (EF), cognitive functioning (CF), social functioning (SF). Three scales assess disease symptoms: fatigue (FA), nausea and vomiting (NV), pain (PA). The main scale assesses global health status (QL). Six questions, interpreted individually, assess disease symptoms: appetite loss (AP), dyspnoea (DY), insomnia (SL), constipation (CO), diarrhoea (DI), financial difficulties (FI) [24].

Interpreting the results in the scope of scales of the functional condition, researchers obtain information on the level of functioning of patients. The number of points obtained on scales assessing disease symptoms indicates the level of influence of ailments on the QoL. The results obtained in the scope of individual questions assessing disease symptoms provide information on the severity of symptoms in the studied patients. The number of points from the scale of the QoL is a measure of subjective feelings and individual assessment of health and QoL by the patient.

Specific questionnaires have been developed to measure the QoL in patients with a specific type/ location of cancer more completely. They constitute an extension and complement to the main tool. They consist of several or several dozen questions that are an integral part of the QLQ-C30 questionnaire. Due to changes in therapeutic profiles introduced over the years, it is necessary to regularly update additional modules so that the questions contained in them adequately relate to the most common problems associated with a specific type of therapy.

EORTC developed specific tools for more than 20 cancer types/locations. Moreover, measure- 
ment tools were constructed for various groups of patients - adolescents and young adults, elderly cancer patients, palliative cancer care patients as well as to measure and assess cancer cachexia, cancer-related fatigue, communication, and satisfaction with in-patient cancer care.

\section{QLQ-BR23 Questionnaire (Quality of Life Questionnaire for Breast Cancer)}

QLQ-BR23 constitutes a complementary module to the QLQ-C30 questionnaire, used to measure the QoL in women with breast cancer at various stages of the disease, treated with chemotherapy, hormone therapy, surgery or radiotherapy. It contains 23 questions, assigned to five scales, two of which assess the functional state - body image (BRBI) and sexual functioning (BRSEF) and three concern symptoms: systemic therapy side effects (BRST), breast symptoms (BRBS) and arm symptoms (BRAS). Additionally, three individual questions relate to sexual enjoyment (BRSEE), future perspective (BRFU) and the fact of being upset by hair loss (BRHL) [25].

The QLQ-BR23 questionnaire was developed in 1996. Since then, modifications have been made to the diagnostic and therapeutic management of breast cancer, and the new treatment methods are associated with other side effects. Therefore, a new 45-item module for assessing the QoL in patients with breast cancer, QLQ-BR45, has been developed. Work on its validation is currently underway [26].

A special group of patients among patients with breast cancer comprises women who have undergone mastectomy and breast reconstruction. EORTC has developed a specific tool to assess the QoL in this group of patients, QLQ-BRECON23, used together with the QLQ-C30 and QLQ-BR23 basic modules. The use of such a research procedure enables the performance of an in-depth assessment of the QoL in patients undergoing mastectomy. The QLQ-BRECON23 module consists of 23 questions, 14 of which relate to the QoL of women before mastectomy and reconstruction, and the remaining 9 items are relevant after reconstruction. 20 items were assigned to 6 scales - breast cosmetic, satisfaction with surgery, donor-site symptoms, nipple cosmetic, sexuality, treatment side-effects - while 3 are of an individual nature: appearance of scars, nipple los, preserve/recon nipple [27].

\section{QLQ-OV28 Questionnaire (Quality of Life Questionnaire for Ovarian Cancer)}

The QLQ-OV28 module was designed as a complement to the QLQ-C30 tool in the study of the QoL of women with ovarian cancer, sur- gically treated with or without chemotherapy. It consists of 28 items, assigned to 8 scales. The tool allows for the assessment of side effects of chemotherapy (including abdominal and gastrointestinal symptoms, peripheral neuropathy), hormonal and menopausal symptoms, attitude to disease/treatment, body image and sexual functioning [28].

\section{QLQ-PR25 Questionnaire (Quality of Life Questionnaire for Prostate Cancer)}

The QLQ-PR25 module was designed to comprehensively measure the QoL in men with localized and metastatic prostate cancer. The results of this 25-item questionnaire are described on 5 scales: bowel symptoms (4 items), urinary symptoms (8 items), hormonal treatment-related symptoms (6 items), sexual activity ( 2 items), sexual functioning ( 6 items). One question concerning incontinence aid functions independently [29].

\section{QLQ-CR29 Questionnaire (Quality of Life Questionnaire for Colorectal Cancer)}

The QLQ-CR29 module is intended to be used as a measurement standard in studies of the QoL of patients with various stages of colorectal cancer, regardless of the treatment method. It is an updated, shorter version of the QLQ-CR38 tool developed in 1999. It consists of 29 questions. The results are described on 6 scales concerning: micturition problems, abdominal and pelvic pain, defaecation problems, faecal incontinence, anxiety, body image. Moreover, 11 individual questions are subject to individual interpretation [30].

\section{QLQ-LC13 Questionnaire (Quality of Life Questionnaire for Lung Cancer)}

The QLQ-LC13 module was designed to be used with the QLQ-C30 basic questionnaire among patients with lung cancer at various stages treated with chemotherapy and/or radiotherapy.

The tool consists of 13 questions relating to the symptoms of the disease and treatment-related side effects. This module includes one multiitem scale assessing the occurrence and severity of dyspnoea and a series of individual questions to identify and assess pain (depending on its location), cough, dysphagia, peripheral neuropathy, alopecia, haemoptysis and concerning the need for painkillers [31].

EORTC is updating the QLQ-LC13 questionnaire, used since 1994, to QLQ-LC29, which, in addition to the original items, includes new ones on side effects of targeted and surgical treatment. It is recommended that the updated module become the standard of QoL assessment in clinical studies on lung cancer [32]. 


\section{Conclusions}

In addition to the aspects that improve health indicators and extend life expectancy, the complex process of restoring the well-being of patients suffering from malignant tumours should also incorporate measures to ensure an appropriate level of QoL. A holistic approach to care for cancer patients allows one to appreciate the subjective dimension related to the individual assessment of a patient's performance in key areas of life. The patient becomes a partner whose active role in the diagnostic and therapeutic process is particularly important.

\section{Conflict of interest}

The authors declare no conflict of interest.

\section{References}

1. Schipper H. Quality of life: principles of the clinical paradigm. J Psychosocial Oncol 1990; 8: 171-85.

2. The WHOQOL Group. The World Health Organization Quality of Life assessment (WHOQOL): position paper from the World Health Organization. Soc Sci Med 1995; 41: 1403-9.

3. Paraskevi T. Quality of Life: Definition and Measurement. Eur J Psychol 2013; 9: 150-62.

4. Chmaj-Wierzchowska K, Rzymski P, Wojciechowska M, Parda I, Wilczak M. Health-related quality of life (Nottingham Health Profile) in patients with endometriomas: correlation with clinical variables and self-reported limitations. Arch Med Sci 2020; 16: 584-91.

5. Allison PJ, Locker D, Feine JS. Quality of life: a dynamic construct. Soc Sci Med 1997; 45: 221-30.

6. Moorey S, Greer S. Psychological therapy for patients with cancer. New York: Oxford: Heinemann Medical Books; 1989.

7. Czerw A, Marek E, Deptała A. The use of the Mini-MAC Scale in the evaluation of mental adjustment to cancer. Contemp Oncol 2015; 19: 414-9.

8. Zucca A, Lambert S, Boyes A, Pallant J. Rasch analysis of the Mini-Mental Adjustment to Cancer Scale (mini$M A C$ ) among a heterogeneous sample of long-term cancer survivors: A cross-sectional study. Health Qual Life Outcomes 2012; 10: 55.

9. World Health Organization. Division of Mental Health and Prevention of Substance Abuse. WHOQOL Measuring Quality of Life. Programme on mental health. Geneva: World Health Organization; 1997.

10. Arora NK. Interacting with cancer patients: the significance of physicians' communication behaviour. Soc Sci Med 2003; 57: 791-806.

11. Prieto L, Sacristan J. Problems and solutions in calculating quality-adjusted life years (QALYs). Health Qual Life Outcomes 2003; 1: 80.

12. Kaplan RM, Anderson JP. The General Health Policy Model: An Integrated Approach. In: Spilker B, editor. Quality of Life and Pharmacoeconomics in Clinical Trials. $2^{\text {nd }}$ ed. Philadelphia: Lippincott-Raven Publishers; 1996. p. 309-22.

13. Chen TH, Li L, Kochen MM. A systematic review: how to choose appropriate health-related quality of life
(HRQOL) measures in routine general practice? J Zhejiang Univ Sci B 2005; 6: 936-40.

14. Lin XJ, Lin IM, Fan SY. Methodological issues in measuring health-related quality of life. Tzu Chi Med J 2013; 25: 8-12.

15. Karnofsky DA, Burchenal JH. The Clinical Evaluation of Chemotherapeutic Agents in Cancer. In: Evaluation of Chemotherapeutic Agents. MacLeod CM, editor. New York: Columbia University Press; 1949: 196.

16. Hays R, Sherbourne C, Mazel R. User's Manual for the Medical Outcomes Study (MOS) Core Measures of Health-Related Quality of Life. Santa Monica: RAND Corporation; 1995

17. Ware J, Kosiński M, Dewey J. How to score version 2 of the SF-36 Health Survey (Standard and Acute Forms). $3^{\text {rd }}$ ed. Lincoln: Quality Metric Incorporated; 2002.

18. World Health Organization. Division of Mental Health. WHOQOL-BREF. Introduction, administration, scoring and generic version of the assessment. Field Trial Version. Geneva: World Health Organization; 1996.

19. The Euro Qol Group. EuroQol - a new facility for the measurement of health-related quality of life. Health Policy 1990; 16: 199-208.

20. Suárez-Llanos J, Vallejo-Torres L, García-Bello M, et al. Cost-effectiveness of the hospital nutrition screening tool CIPA. Arch Med Sci 2020; 16: 273-81.

21. Szende A, Williams A. Measuring Self-Reported Population Health: An International Perspective based on EQ-5D. Rotterdam: EuroQol Group; 2004.

22. Hunt SM, McEwen J, McKenna SP. Measuring health status: a new tool for clinicians and epidemiologists. J R Coll Gen Pract 1985; 35: 185-8.

23. Spitzer WO, Dobson AJ, Hall J, Chesterman E, Levi J, Shepherd R. Measuring the quality of life of cancer patients: a concise QL-Index for use by physicians. J Chronic Dis 1981; 34: 585-97.

24. Aaronson NK, Ahmedzai S, Bergman B, et al. The European Organisation for Research and Treatment of Cancer QLQ-C30: a quality-of-life instrument for use in international clinical trials in oncology. J Natl Cancer Inst 1993; 85: 365-76.

25. Sprangers MA, Groenvold M, Arraras JI, et al. The European Organization for Research and Treatment of Cancer breast cancer-specific quality-of-life questionnaire module: first results from a three-country field study. J Clin Oncol 1996; 14: 2756-68.

26. Bjelic-Radisic V, Cardoso F, Cameron D, et al. EORTC Quality of Life Group and Breast Cancer Group. An international update of the EORTC questionnaire for assessing quality of life in breast cancer patients: EORTC QLQ-BR45. Ann Oncol 2020: 31: 283-8.

27. Winters ZE, Afzal M, Rutherford C, et al. European Organisation for Research and Treatment of Cancer Quality of Life Group. International validation of the European Organisation for Research and Treatment of Cancer QLQ-BRECON23 quality-of-life questionnaire for women undergoing breast reconstruction. Br J Surg 2018; 105 209-22.

28. Greimel E, Bottomley A, Cull A, et al. EORTC Quality of Life Group and the Quality of Life Unit. An international field study of the reliability and validity of a disease-specific questionnaire module (the QLQ-OV28) in assessing the quality of life of patients with ovarian cancer. Eur J Cancer 2003; 39: 1402-8.

29. van Andel G, Bottomley A, Fosså SD, et al. An international field study of the EORTC QLQ-PR25: a questionnaire for assessing the health-related quality of life 
of patients with prostate cancer. Eur J Cancer 2008; 44:

2418-24.

30. Gujral S, Conroy T, Fleissner C, et al. European Organisation for Research and Treatment of Cancer Quality of Life Group. Assessing quality of life in patients with colorectal cancer: an update of the EORTC quality of life questionnaire. Eur J Cancer 2007; 43: 1564-73.

31. Bergman B, Aaronson NK, Ahmedzai S, Kaasa S, Sullivan M. The EORTC QLQ-LC13: a modular supplement to the EORTC Core Quality of Life Questionnaire (QLQ-C30) for use in lung cancer clinical trials. EORTC Study Group on Quality of Life. Eur J Cancer 1994; 30A: 635-42.

32. Koller M, Hjermstad MJ, Tomaszewski KA, et al. An international study to revise the EORTC questionnaire for assessing quality of life in lung cancer patients. Ann Oncol 2017; 28: 2874-81. 